Revue d'histoire de l'Amérique française

\title{
Dynamique des populations locales : la formation des paroisses rurales au Saguenay (1840-1911)
}

\section{Gérard Bouchard et Jeannette Larouche}

Volume 41, numéro 3, hiver 1988

URI : https://id.erudit.org/iderudit/304583ar

DOI : https://doi.org/10.7202/304583ar

Aller au sommaire du numéro

\section{Éditeur(s)}

Institut d'histoire de l'Amérique française

\section{ISSN}

0035-2357 (imprimé)

1492-1383 (numérique)

Découvrir la revue

\section{Citer cet article}

Bouchard, G. \& Larouche, J. (1988). Dynamique des populations locales : la formation des paroisses rurales au Saguenay (1840-1911). Revue d'histoire de l'Amérique française, 41(3), 363-388. https://doi.org/10.7202/304583ar
Résumé de l'article

Cette étude part du principe que le contexte dans lequel une société se constitue lui confère des traits durables et éclaire son évolution ultérieure. Dans cet esprit, les auteurs ont entrepris une étude micro-locale de la formation de la population du Saguenay, à partir de 1838. Dans une première étape de la recherche, dix paroisses sont prises à témoin. On y observe les lieux de provenance, le calendrier d'implantation, les caractéristiques sociales et familiales des pionniers.

L'étude met en relief l'importance de l'encadrement familial et la très grande homogénéité de la population fondatrice. Sous ce rapport l'expérience saguenayenne semble s'apparenter à celle de la plupart des régions de colonisation du Québec. Par contre, elle contraste violemment avec ce que l'on sait de la "frontière " canadienne-anglaise et américaine du $19^{\mathrm{e}}$ siècle. Ce contraste dessine en même temps deux problématiques également opposées : on s'interroge, dans le premier cas, sur les conditions d'une éventuelle différenciation collective, et dans le deuxième cas, sur les modes de construction d'un « consensus » ou d'une cohésion socio-culturelle. 


\title{
DYNAMIQUE DES POPULATIONS LOCALES: LA FORMATION DES PAROISSES RURALES AU SAGUENAY (1840-1911) ${ }^{1}$
}

\author{
GÉRARD BOUCHARD \\ JEANNETTE LAROUCHE \\ Centre SOREP \\ Université du Québec à Chicoutimi
}

\section{RÉSUMÉ}

Cette étude part du principe que le contexte dans lequel une société se constitue lui confère des traits durables et éclaire son évolution ultérieure. Dans cet esprit, les auteurs ont entrepris une étude micro-locale de la formation de la population du Saguenay, à partir de 1838. Dans une première étape de la recherche, dix paroisses sont prises à témoin. On y observe les lieux de provenance, le calendrier d'implantation, les caractéristiques sociales et familiales des pionniers.

L'étude met en relief l'importance de l'encadrement familial et la très grande homogénéité de la population fondatrice. Sous ce rapport l'expérience saguenayenne semble s'apparenter à celle de la plupart des régions de colonisation du Québec. Par contre, elle contraste violemment avec ce que l'on sait de la «frontière» canadienne-anglaise et américaine du $19 \mathrm{e}$ siècle. Ce contraste dessine en même temps deux problématiques également opposées: on s'interroge, dans le premier cas, sur les conditions d'une éventuelle différenciation collective, et dans le deuxième cas, sur les modes de construction d'un «consensus» ou d'une cohésion socio-culturelle.

\section{ABSTRACT}

This study starts with the idea that the context in which a society is constituted gives it lasting characteristics and helps explain its later evolution. With this perspective, the authors undertook a micro-local study of the formation of the Saguenay population, beginning in 1838. In the first stage of the research project, ten parishes were taken as evidence. Their pioneers' places of origin, settlement history, and social and family characteristics were observed.

The study emphasizes the importance of family ties and the very great homogeneity of the founding population. In this regard, the Saguenay case seems to be similar to those of most of the colonized regions of Quebec. On the other hand, it contrasts markedly with what is known about the nineteenth century English-Canadian and American «frontier». This contrast points to two opposite problems at the same time: in the first case, there is the question of the conditions of a possible collective differentiation, and, in the second, the major question is how to build a "consensus» or some kind of socio-cultural cohesion.

1 Nos travaux ont pu être réalisés grâce à l'appui financier du Conseil de recherches en sciences humaines du Canada (Ottawa) et du Fonds FCAR (Québec). Alain Simard et Danielle Lavoie ont aussi collaboré à l'élaboration des données, de même que Marcel Thivierge, généalogiste. 
La présente enquête porte sur la région du Saguenay depuis son ouverture au peuplement blanc, vers 1840 , jusqu'au début du 20 e siècle. Cette période correspond à l'immigration pionnière qui a assuré l'occupation du sol dans le Bas et le Haut-Saguenay - la mise en valeur de l'écoumène dans la sous-région du Lac Saint-Jean a été complétée vers 1940 (Carte). Les questions abordées ici ont trait à la colonisation dans un cadre régional, l'accent étant mis sur les dimensions démographique, sociale et spatiale du peuplement. Nous voulons éclairer en particulier a) le calendrier et les modalités de mise en place de la population pionnière, b) l'arrière-plan géographique et culturel des immigrants, c) certains paramètres socio-économiques des mouvements migratoires. À long terme, il s'agit de reconstituer la dynamique des populations rurales, ce concept servant à désigner l'ensemble des facteurs et comportements collectifs - démographiques certes, mais aussi culturels, économiques et sociaux -- qui déterminent la formation et la reproduction physique d'une population, et règlent son évolution dans un cadre spatio-temporel donné.

Sur un plan plus général, nous voulons situer la colonisation saguenayenne dans le contexte canadien et américain, de manière à en dégager les spécificités. Cette perspective comparative est facilitée par le nombre grandissant d'études monographiques qui, depuis une quinzaine d'années, ont contribué à renouveler les frontier studies en les

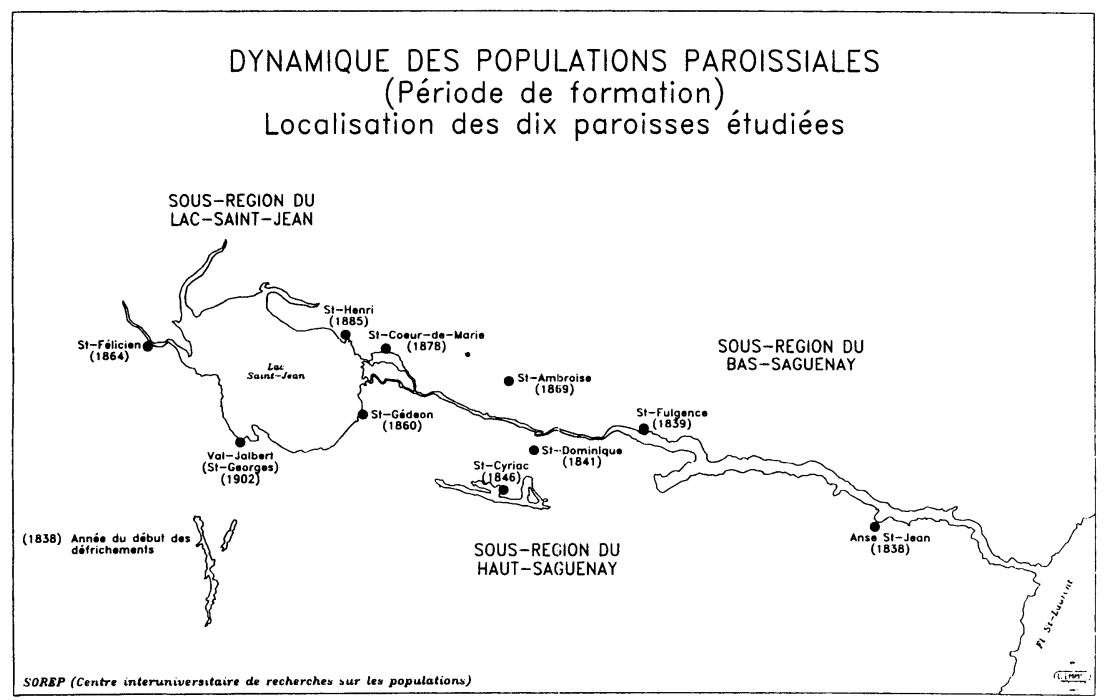


ouvrant aux préoccupations et aux méthodes de l'histoire sociale ${ }^{2}$. Ces études font une large place aux nouvelles formes de vie collective associées au contexte de peuplement, aux inégalités socio-économiques, aux continuités et aux ruptures culturelles, aux paramètres physiques et économiques de la reproduction familiale et de la croissance démographique.

Ce type de préoccupation a inspiré la recherche dont nous présentons ici une première tranche. Travaillant sur un échantillon de dix paroisses $^{3}$, nous avons centré l'analyse sur la période de formation, celle qui va de l'arrivée du premier colon à l'ouverture officielle de la

2 Nous nous limiterons à quelques repères bibliographiques. Pour le Québec, voir Louise Dechêne, Habitants et marchands de Montréal au XVIIe siècle (Paris, Plon, coll. «Civilisation et mentalités», 1974), 581 p.; Jacques Mathieu, François Béland et al., «Peuplement colonisateur au XVIIIe siècle dans le gouvernement du Québec», in R. L. Emerson, W. Kinsley, W. Moser, dir., L'homme et la nature, Actes de la Société canadienne d'étude du dix-huitième siècle, II: 127-138; Serge Courville, «Espace, territoire et culture en Nouvelle-France: une vision géographique», Revue d'histoire de l'Amérique française, 37,3 (décembre 1983): 417-430; Normand Séguin, La conquête du sol au 19e siècle (Québec, Éditions du Boréal Express, 1977), 295 p.; J. I. Little, «The Parish and Economic Development of Settlers in Two Quebec Townships, 18511870», Canadian Papers in Rural History, 1: 89-113; Yves Beauregard, Serge Goudreau et al., «Famille, parenté et colonisation en Nouvelle-France», Revue d' histoire de l'Amérique française, 39,3 (hiver 1986): 391-405. Pour le Canada anglais, voir David Gagan, Hopeful Travellers: Families, Land, and Social Change in Mid-Victorian Peel County, Canada West (Toronto, University of Toronto Press, 1981), 197 p.; John J. Mannion, W. G. Handcock and al., The Peopling of Newfoundland. Essays in Historical Geography (John J. Mannion, ed., Memorial University of Newfoundland, 1977), 289 p.; Darrel A. Norris, «Migration, Pioneer Settlement, and the Life Course: the First Families of an Ontario Township», in Donald H. Akenson, ed., Canadian Papers in Rural History (Gananoque, Langdale Press, 1984), 4: 130-152; Paul Voisey, Vulcan. The Making of a Prairie Community (Alberta, 1987), 384 p.; Glenn J. Lockwood, «Irish Immigrants and the «Critical Years», in Eastern Ontario: the Case of Montague Township», in Donald H. Akenson, ed., Canadian Papers in Rural History (Gaganoque, Langdale Press, 1984), 4: 153178. Pour les États-Unis, voir John C. Hudson, «Migration to an American Frontier», Annals of the Association of American Geographers, 66,2 (June 1976): 242-265; «Theory and Methodology in Comparative Frontier Studies», in David Harry Miller and Jerome O. Steffen, eds., The Frontier: Comparative Studies (University of Oklahoma Press, 1977), 11-31; Kenneth Lockridge, A New England Town, the First Hundred Years: Dedham, Massachusetts, 1636-1736 (New York, Norton, 1970); Phillip Greven, Four Generations: Populations, Land and Family in Colonial Andover (Ithaca, New York, Cornell University Press, 1970); Hal S. Barron, Those who Stayed Behind. Rural Society in Nineteenth Century New England (Cambridge, Cambridge University Press, 1984), 184 p.; Jon Gjerde, «The Effects of Community on Migration: Three Minnesota Townships 1885-1905», Journal of Historical Geography, 5 (1979): 403-422; John Rice, «The Role of Culture and Community in Frontier Prairie Farming», Journal of Historical Geography, 3 (1977): 155-175.

3 Choisies en fonction de divers critères: elles devaient être dispersées dans l'ensemble de la région et sur toute la période étudiée; elles ne devaient pas avoir été divisées avant 1911; elles devaient refléter différents niveaux de richesse et il fallait disposer, pour chacune d'elles, de sources documentaires suffisamment riches et variées (voir Carte). Le fichier-réseau de SOREP a été la principale source utilisée, mais la recherche a aussi fait largement appel aux recensements nominatifs du gouvernement canadien et des paroisses («livres des âmes»), au Greffe des arpentages du ministère de l'Énergie et des Ressources (Québec), à des listes de paroissiens (pétitions, etc.), à des monographies de paroisses et à divers dossiers du fonds Victor-Tremblay aux Archives nationales du Québec à Chicoutimi. L'apport combiné de ces sources - surtout les fiches de familles reconstituées et les diverses listes nominatives - a permis des recoupements grâce auxquels il a été possible d'identifier la plupart des pionniers et de déterminer le moment de leur établissement. 
paroisse, c'est-à-dire la date d'installation du premier curét. À cet égard, il est utile de rappeler le contexte régional dans lequel s'insère la présente étude. La région du Saguenay a été ouverte à la colonisation vers le milieu du $19 \mathrm{e}$ siècle, dans le même courant qui a conduit à la création de la plupart des régions dites périphériques au Québec ${ }^{5}$. Sa population s'est accrue rapidement, surtout grâce aux très forts excédents de son mouvement naturel (taux supérieurs à 30 0/00 jusqu'en 1960). De 4801 en 1850, le nombre des habitants est passé à 100000 en 1930 et à près de 300000 en 1985. L'âge au mariage y était bas et la fécondité exceptionnellement élevée, supérieure même à celle des Huttérites ${ }^{6}$. Le mouvement de peuplement - ou d'occupation du sol — s'est étendu sur un siècle environ (1840-1940); il a donné naissance à une dizaine de petites villes et à une soixantaine de paroisses rurales. Il a progressé d'une manière presque linéaire, d'est en ouest, contournant le lac Saint-Jean dans le sens des aiguilles d'une montre ${ }^{7}$.

\section{1 - MISE EN PLACE DE LA PREMIÈRE GÉNÉRATION DE PIONNIERS}

Il importe d'abord de rendre compte du rythme de formation des paroisses, du calendrier d'arrivée des immigrants à l'échelle locale et des modalités de recrutement migratoire.

\section{A - Durée de la période pionnière}

On relève d'abord que les paroisses les plus récemment ouvertes se développent plus rapidement que les anciennes, ce qui se vérifie en particulier pour Saint-Coeur-de-Marie, Saint-Henri-de-Taillon et ValJalbert (Tableau 1). Ces paroisses sont toutes localisées à l'ouest de la région du Saguenay. L'explication paraît se trouver dans le fait que ces paroisses, contrairement aux anciennes, ont bénéficié d'un double apport migratoire. D'abord une immigration de l'extérieur de la région, qui s'est poursuivie jusque dans le 20e siècle, et ensuite une immigration de l'intérieur qui était alimentée par le débordement des vieilles paroisses saguenayennes situées plus à l'est. Le tableau 2 confirme ce phénomène. On observe que, sur l'ensemble des paroisses ouvertes, celles

\footnotetext{
4 Ce critère d'ordre administratif constitue un repère socio-économique pertinent dans la mesure où la nomination du premier curé faisait suite à une requête des paroissiens dans laquelle ils devaient faire état du développement des terres. Il est évident en effet que l'évêque ne pouvait assigner un curé permanent à la nouvelle paroisse que dans la mesure où ses habitants étaient capables de subvenir à ses besoins et à ceux du culte. Toutes les données présentées ici sont circonscrites à ce premier volet de la période de formation des paroisses.

5 Raoul Blanchard, Le Canada français. Province de Québec (Paris, Montréal, 1960), 316 p.

Christian Pouyez, Yolande Lavoie, Gérard Bouchard et al., Les Saguenayens. Introduction à l'histoire des populations du Saguenay XVI-XXe siècles (Québec, Presses de l'Université du Québec, 1983), 396 p. Voir le chapitre 6.

7 Gérard Bouchard, «Le peuplement blanc», in ibid., chapitre 4.
} 
TABLEAU 1

Dynamique des populations paroissiales

(I: Période de formation)

Durée de la période pionnière, selon les paroisses

\begin{tabular}{|c|c|c|c|c|}
\hline $\begin{array}{c}\text { Paroisses } \\
\text { étudiées }\end{array}$ & $\begin{array}{l}\text { Début de la colonisation } \\
\text { (date d'arrivée des } \\
\text { premiers colons) }\end{array}$ & $\begin{array}{l}\text { Année d'ouverture des } \\
\text { registres paroissiaux } \\
\text { (baptêmes, mariages, } \\
\text { sépultures) }\end{array}$ & $\begin{array}{l}\text { (3) } \\
\text { Arrivée du premier curé } \\
\text { dans la paroisse }\end{array}$ & $\begin{array}{c}\text { (4) } \\
\text { Délai entre } \\
\text { (1) et (2) } \\
\text { (en années) }\end{array}$ \\
\hline $\begin{array}{l}\text { Anse St-Jean } \\
\text { St-Fulgence } \\
\text { St-Dominique } \\
\text { St-Gédéon } \\
\text { St-Félicien } \\
\text { St-Cyriac } \\
\text { St-Ambroise } \\
\text { St-Coeur-de-Marie } \\
\text { St-Henri-de-Taillon } \\
\text { Val-Jalbert }\end{array}$ & $\begin{array}{l}1838 \\
1839 \\
1841 \\
1860 \\
1864 \\
1864 \\
1869 \\
1879 \\
1885 \\
1902\end{array}$ & $\begin{array}{l}06 \text { octobre } 1861 \\
26 \text { février } 1871 \\
10 \text { octobre } 1866 \\
05 \text { janvier } 1881 \\
06 \text { janvier } 1884 \\
11 \text { janvier } 1885 \\
14 \text { décembre } 1903 \\
11 \text { octobre } 1889 \\
06 \text { octobre } 1902 \\
30 \text { septembre } 1911\end{array}$ & $\begin{array}{l}28 \text { septembre } 1861 \\
20 \text { octobre } 1871 \\
\text { octobre } 1866 \\
23 \text { août } 1880 \\
19 \text { août } 1883 \\
\text { septembre } 1889 \\
01 \text { septembre } 1903 \\
27 \text { août } 1889 \\
26 \text { août } 1902 \\
\text { septembre } 1911\end{array}$ & $\begin{array}{r}23 \\
32 \\
25 \\
21 \\
20 \\
21 \\
34 \\
10 \\
17 \\
9\end{array}$ \\
\hline
\end{tabular}

Note: Délai moyen entre (1) et (2): 21 ans 2 mois. (SOREP) 
TABLEAU 2

Durée de la période pionnière: ensemble des paroisses créées avant 1911, regroupées en sous-régions ${ }^{\mathrm{a}}$

\begin{tabular}{|c|c|c|c|c|}
\hline \multirow{2}{*}{$\begin{array}{c}\text { Délais } \\
\text { (en années) }\end{array}$} & Bas-Saguenay & Haut-Saguenay & Lac Saint-Jean & \multirow{2}{*}{ Région } \\
\cline { 2 - 5 } & - & 2 & 3 & 5 \\
$6-5$ & - & 1 & 6 & 7 \\
$11-15$ & - & - & 6 & 6 \\
$16-20$ & 1 & 2 & 1 & 4 \\
$21-25$ & 1 & - & - & 2 \\
$26-30$ & 1 & 1 & 1 & 2 \\
$31-35$ & 27 & 20,3 & 14,6 & 17 \\
\hline 35 et plus & 3 & 10 & 26 & 39 \\
\hline Délai moyen & - & 1 & & 2 \\
\hline Nombre de & & & & \\
\hline
\end{tabular}

(a) Cette durée correspond à la période pendant laquelle le nouvel établissement a eu le statut de mission. (SOREP) 
TABLEAU 3

Rythme de formation et taille de la population des paroisses au moment de leur création

\begin{tabular}{|l|c|c|c|}
\hline \multicolumn{1}{|c|}{$\begin{array}{c}\text { Paroisses } \\
\text { étudiées }\end{array}$} & $\begin{array}{c}(1) \\
\text { Durée de la } \\
\end{array}$ & $\begin{array}{c}\text { (2) } \\
\text { période pionnière } \\
\text { (années) }\end{array}$ & $\begin{array}{c}\text { Population de la } \\
\text { paroisse au moment } \\
\text { de sa création }\end{array}$ \\
\hline (ouverture des registres)
\end{tabular}

(SOREP) 
qui sont situées dans la sous-région du Lac Saint-Jean, c'est-à-dire la région la plus occidentale du Saguenay, se sont ouvertes plus rapidement, la durée moyenne de la période pionnière y étant de 14,6 années contre 20,3 et 27 pour les deux autres sous-régions. Enfin, il ne semble pas que la durée de la période pionnière soit directement reliée au niveau de la population atteinte, une fois franchi un seuil critique. Le tableau 3 montre en effet qu'il n'y a pas de corrélation entre ces deux variables. On devine ici que plusieurs facteurs pouvaient retarder la décision épiscopale d'assigner ou non un curé à un nouvel établissement. On pense ici en particulier à la disponibilité des prêtres et à diverses considérations de politique locale.

\section{$B$ - Calendrier d'arrivée des immigrants}

La statistique du nombre annuel d'arrivants dans chaque paroisse n'a pas permis de faire ressortir un modèle dominant. Dans quelques cas seulement, on relève une hausse dans le nombre d'arrivants pour les années qui précèdent immédiatement la création officielle de la paroisse. Pour le reste, on observe une grande diversité, hormis le fait que presque partout les arrivées se faisaient par à-coups, comme si elles obéissaient à des conjonctures locales, à l'échelle familiale (Graphique 1). Il s'avère par ailleurs (Tableau 4) que les plus vieilles paroisses accueillaient surtout des familles dont le mariage avait été célébré à l'extérieur de la région tandis que les paroisses plus récentes accueillaient davantage de couples formés dans une paroisse saguenayenne créée antérieurement. Ce résultat atteste encore une fois le mouvement de déplacement ou de débordement des vieilles paroisses de l'est du Saguenay vers celles de l'ouest, mouvement qui affectait surtout les familles pionnières les plus anciennes ou leurs descendants. Enfin, et comme on s'y attendait, le tableau 5 confirme que les plus vieilles paroisses constituaient le premier point de chute des immigrants. On relève une exception pour Saint-Ambroise, créée par une immigration ad hoc suscitée par des promoteurs de la colonisation.

\section{C - Modalités du recrutement migratoire}

En moyenne, les migrants intra-régionaux franchissaient 31 kilomètres pour venir s'établir dans une nouvelle paroisse. Mais ce chiffre dissimule une disparité importante (Tableau 6). Dans 2 cas sur 3, on se déplaçait sur une distance variant entre 10 et 40 kilomètres. Les paroisses plus isolées, comme Anse-Saint-Jean et Saint-Félicien, commandaient des migrations plus longues, et au sein d'une même paroisse, on observe des réalités contrastées. En fait, on est en présence de deux mouvements superposés; l'un est un mouvement de peuplement de proche en proche, l'autre fait des bonds d'une sous-région à l'autre. 
DYNAMIQUE DES POPULATIONS LOCALES...

Grophique 1

Calendrier d'arrivée des immigrants par paroisse durant la période pionnière

(Nombre d'individus)
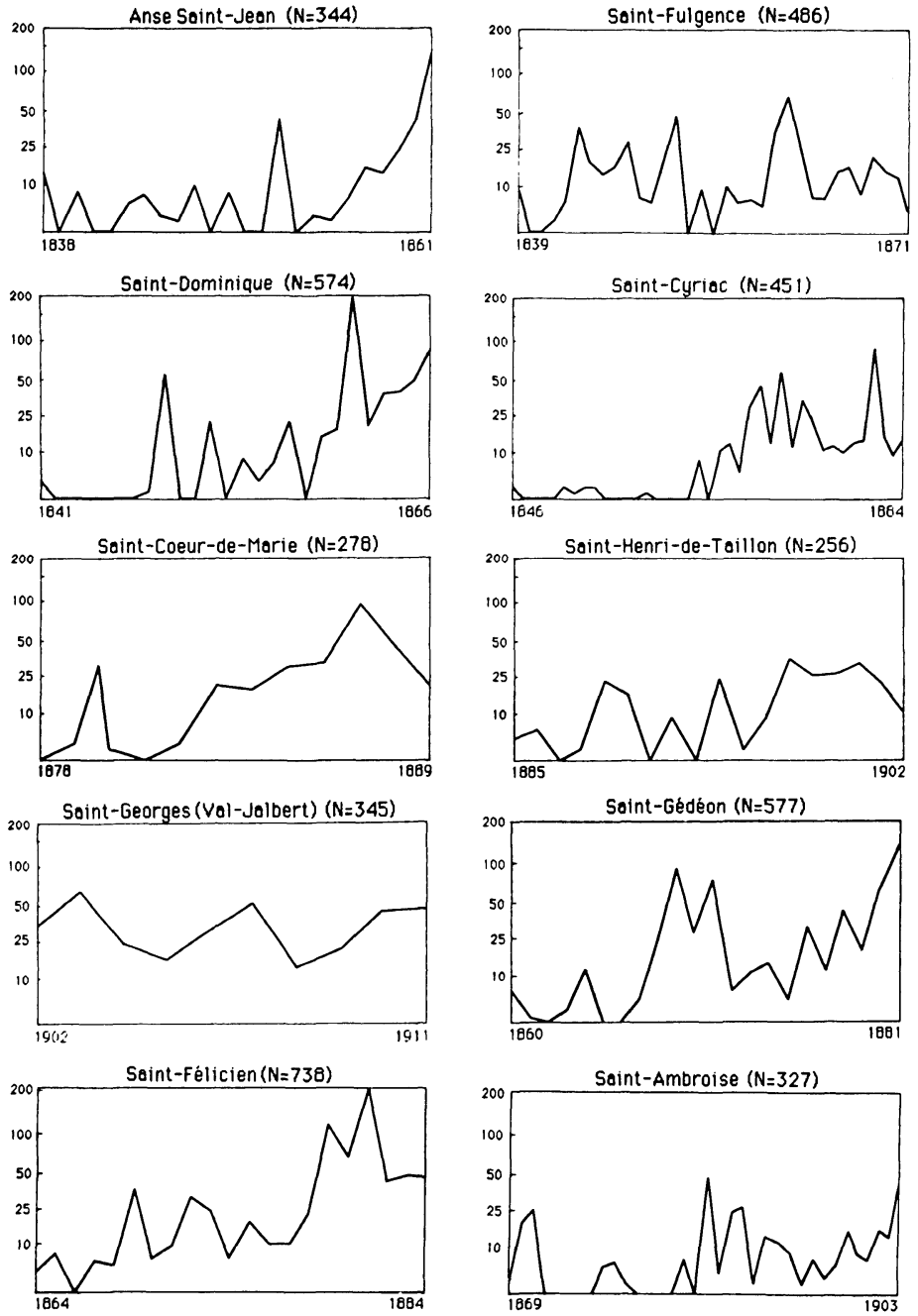

Note $N=$ Nombre totol dimmigrents durent lo periode SOREP 
TABLEAU 4

Répartition des familles de pionniers selon que les parents se sont mariés au Saguenay ou à l'extérieur

\begin{tabular}{|l|c|c|c|c|c|c|}
\hline \multicolumn{1}{|c|}{$\begin{array}{c}\text { Paroisses } \\
\text { étudiées }\end{array}$} & \multicolumn{2}{c|}{$\begin{array}{c}\text { Proportion des mariages célébrés } \\
\text { à l'extérieur de la région }\end{array}$} & $\begin{array}{c}\text { Proportion des mariages célébrés } \\
\text { à l'intérieur de la région }\end{array}$ & \multicolumn{3}{c|}{ TOTAL } \\
\hline (par ordre d'ancienneté) & NA & $\%$ & NA & $\%$ & NA & $\%$ \\
\hline Anse St-Jean & 63 & 80,8 & 15 & 19,2 & 78 & 100 \\
St-Fulgence & 73 & 52,5 & 66 & 47,5 & 139 & 100 \\
St-Dominique & 84 & 50,6 & 82 & 49,4 & 166 & 100 \\
St-Gédéon & 71 & 50,4 & 70 & 49,6 & 141 & 100 \\
St-Félicien & 91 & 50,0 & 91 & 50,0 & 182 & 100 \\
St-Cyriac & 21 & 21,9 & 75 & 78,1 & 96 & 100 \\
St-Ambroise & 18 & 22,0 & 64 & 78,0 & 82 & 100 \\
St-Coeur-de-Marie & 6 & 11,1 & 48 & 88,9 & 54 & 100 \\
St-Henri-de-Taillon & 19 & 27,1 & 51 & 72,9 & 70 & 100 \\
Val-Jalbert & 25 & 26,9 & 68 & 73,1 & 93 & 100 \\
\hline TOTAL & 471 & 42,8 & 630 & 57,2 & 1101 & 100 \\
\hline
\end{tabular}


TABLEAU 5

Itinéraire des familles pionnières formées à l'extérieur du Saguenay

\begin{tabular}{|c|c|c|c|c|c|c|}
\hline \multirow{2}{*}{$\begin{array}{c}\begin{array}{c}\text { Paroisses } \\
\text { étudiées }\end{array} \\
\text { (par ordre d'ancienneté) }\end{array}$} & \multicolumn{2}{|c|}{$\begin{array}{l}\text { Venues directement } \\
\text { dans la paroisse }\end{array}$} & \multicolumn{2}{|c|}{$\begin{array}{l}\text { Se sont d'abord établies dans } \\
\text { une autre paroisse du Saguenay }\end{array}$} & \multicolumn{2}{|c|}{ TOTAL $^{a}$} \\
\hline & NA & $\%$ & NA & $\%$ & NA & $\%$ \\
\hline Anse St-Jean & 53 & 86,9 & 8 & 13,1 & 61 & 100 \\
\hline St-Fulgence & 49 & 70,0 & 21 & 30,0 & 70 & 100 \\
\hline St-Dominique & 45 & 54,2 & 38 & 45,8 & 83 & 100 \\
\hline St-Gédéon & 42 & 59,2 & 29 & 40,8 & 71 & 100 \\
\hline St-Félicien & 51 & 56,0 & 40 & 44,0 & 91 & 100 \\
\hline St-Cyriac & 6 & 37,5 & 10 & 62,5 & 16 & 100 \\
\hline St-Ambroise & 15 & 83,3 & 3 & 16,7 & 18 & 100 \\
\hline St-Coeur-de-Marie & 1 & 16,7 & 5 & 31,3 & 6 & 100 \\
\hline St-Henri-de-Taillon & 7 & 38,9 & 11 & 61,1 & 18 & 100 \\
\hline Val-Jalbert & 10 & 43,5 & 13 & 56,5 & 23 & 100 \\
\hline TOTAL & 279 & 61,1 & 178 & 38,9 & 457 & 100 \\
\hline
\end{tabular}

(a) 13 cas n'on pu être intégrés à ce tableau; ce sont des familles qui, après s'être établies dans la région, l'ont quittée pour y revenir plus tard. (SOREP) 
TABLEAU 6

Distances entre les lieux de provenance des pionniers mariés au Saguenay et la paroisse de colonisation

Paroisses étudiées

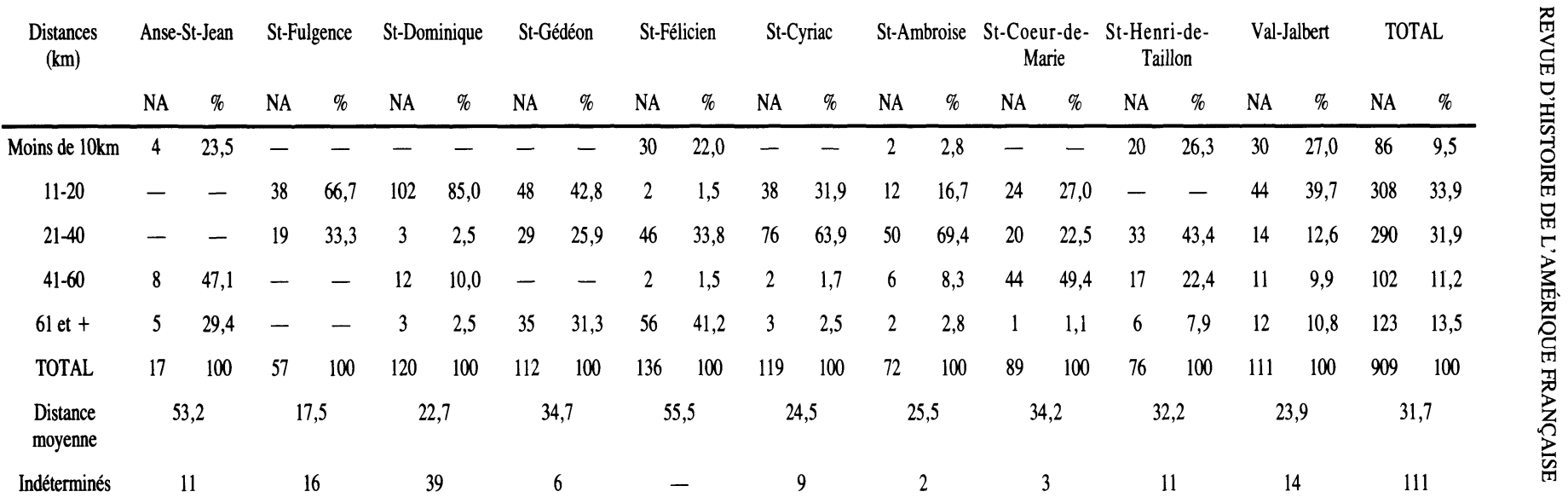

Notes: a) Dans 111 cas (les «Indéterminés»), la résidence des conjoints au mariage n’était pas précisée. b) Dans 11 autres cas, la conjointe résidait déjà dans la paroisse de colonisation. (SOREP) 


\section{TABLEAU 7}

\section{Distribution des lieux d'origine des pionniers dans la région, selon l'importance de leur contribution}

\begin{tabular}{|c|c|c|c|c|c|c|c|c|c|c|c|c|c|c|c|c|c|c|c|c|c|c|}
\hline \multirow{2}{*}{$\begin{array}{l}\text { Pourcentage de } \\
\text { pionniers fournis } \\
\text { par les paroisses }\end{array}$} & \multicolumn{2}{|c|}{ Anse-St-Jean } & \multicolumn{2}{|c|}{ St-Fulgence } & \multicolumn{2}{|c|}{ St-Dominique } & \multicolumn{2}{|c|}{ St-Gédéon } & \multicolumn{2}{|c|}{ St-Félicien } & \multicolumn{2}{|c|}{ St-Cyriac } & \multicolumn{2}{|c|}{ St-Ambroise } & \multicolumn{2}{|c|}{$\begin{array}{c}\text { St-Coeur-de- } \\
\text { Marie }\end{array}$} & \multicolumn{2}{|c|}{$\begin{array}{c}\text { St-Henri-de- } \\
\text { Taillon }\end{array}$} & \multicolumn{2}{|c|}{ Val-Jalbert } & \multicolumn{2}{|c|}{ TOTAL } \\
\hline & NA & $\%$ & $\mathrm{NA}$ & $\%$ & $\mathrm{NA}$ & $\%$ & NA & $\%$ & NA & $\%$ & NA & $\%$ & $\mathrm{NA}$ & $\%$ & $\mathrm{NA}$ & $\%$ & $\mathrm{NA}$ & $\%$ & $\mathrm{NA}$ & $\%$ & NA & $\%$ \\
\hline $\begin{array}{l}\text { En provenance } \\
\text { de la paroisse qui } \\
\text { a le plus } \\
\text { contribué }\end{array}$ & 4 & 23,5 & 34 & 59,6 & 85 & 70,8 & 48 & 42,9 & 38 & 27,9 & 42 & 35,3 & 37 & 51,8 & 26 & 29,2 & 21 & 27,6 & 41 & 36,9 & 376 & 44,5 \\
\hline $\begin{array}{l}\text { En provenance } \\
\text { des deux } \\
\text { premières } \\
\text { paroisses }\end{array}$ & 8 & 47,0 & 42 & 73,7 & 101 & 84,2 & 68 & 60,7 & 68 & 50,0 & 80 & 67,2 & 50 & 69,4 & 50 & 56,2 & 41 & 53,9 & 71 & 64,0 & 579 & 68,5 \\
\hline $\begin{array}{l}\text { En provenance } \\
\text { des trois } \\
\text { premières } \\
\text { paroisses }\end{array}$ & 12 & 70,5 & 49 & 86,0 & 108 & 90,0 & 78 & 69,6 & 85 & 62,5 & 94 & 79,0 & 57 & 79,2 & 62 & 69,7 & 51 & 67,1 & 77 & 69,4 & 673 & 79,6 \\
\hline $\begin{array}{l}\text { En provenance } \\
\text { des quatre } \\
\text { premières } \\
\text { paroisses }\end{array}$ & 16 & 94,1 & 53 & 93,0 & 111 & 92,5 & 88 & 78,6 & 99 & 72,8 & 102 & 85,7 & 63 & 87,5 & 73 & 82,0 & 60 & 78,9 & 83 & 74,8 & 748 & 88,5 \\
\hline $\begin{array}{l}\text { En provenance } \\
\text { des cinq } \\
\text { premières } \\
\text { paroisses }\end{array}$ & 17 & 100 & 57 & 100 & 114 & 95,0 & 95 & 84,8 & 107 & 78,7 & 108 & 90,8 & 68 & 94,4 & 78 & 87,6 & 64 & 84,2 & 87 & 78,4 & 795 & 94,1 \\
\hline $\begin{array}{l}\text { Nombre total de } \\
\text { paroisses ayant } \\
\text { contribué }\end{array}$ & & 5 & & & & & & & & & & 0 & & & & & & & & 5 & & 4 \\
\hline
\end{tabular}


Nous avons cherché à rendre compte de ces déplacements, cette fois du point de la nouvelle paroisse qui se forme, en évaluant la contribution des paroisses plus anciennes (Tableau 7). Dans l'ensemble, près de $70 \%$ des immigrants étaient fournis par deux paroisses seulement; et il n'en fallait pas plus de cinq pour rendre compte de la quasitotalité $(94,1 \%)$ de la nouvelle population. Ces chiffres attestent l'existence de filières migratoires assez exclusives entre certaines paroisses.

Les données dont nous avons fait état jusqu'ici, en rapport avec la mise en place des pionniers, suggèrent que l'expérience saguenayenne s'apparente à celle des autres régions du Québec - dans la mesure où les données sont disponibles, bien sûr. Le cas qui se prête le mieux à la comparaison est celui du Bas-Saint-Laurent, présenté par Alain Laberge ${ }^{8}$. Étudiant trois seigneuries, l'auteur montre que le peuplement pionnier n'était pas le fait d'une immigration ou implantation ponctuelle; c'était plutôt un courant qui s'étirait dans le temps et qui faisait place à des échecs, à un remplacement, tout comme au Saguenay encore une fois. Quant au mode sélectif de recrutement migratoire en fonction de certaines paroisses, on le retrouve également en Nouvelle-France, autour de Québec ${ }^{9}$ et de Trois-Rivières ${ }^{10}$. Par ailleurs, tout rapprochement avec la «frontière» du centre et de l'ouest américain doit s'entourer de précautions, tant les effectifs migratoires impliqués diffèrent. On traite ici, à l'échelle régionale, non pas avec quelques centaines de personnes mais avec des dizaines de milliers ${ }^{11}$. Le calendrier d'implantation est accéléré, le recrutement migratoire est plus complexe et, surtout, l'arrière-plan culturel est beaucoup plus diversifié, comme il ressort des données suivantes.

\section{2 - PROFIL ETHNIQUE ET CULTUREL DES IMMIGRANTS}

Une très grande homogénéité caractérisait le flux migratoire vers le Saguenay. Pratiquement tous les immigrants (plus de 99\%) étaient de langue française et déclaraient appartenir à la religion catholique. Si certains pionniers venus de Charlevoix portaient des noms de famille anglo-saxons (Balckburn, Warren, Harvey...), c'est qu'ils descendaient de familles écossaises établies dans Charlevoix au 18e siècle puis rapidement assimilées à la culture francophone.

\footnotetext{
8 Alain Laberge, «Aspects de la mobilité dans trois seigneuries de la Côte-du-Sud sous le Régime français: les soldes migratoires dans la Grande Anse (1672-1752)». Communication présentée à la réunion annuelle de la Société historique du Canada, à Hamilton, en 1987.

9 Yves Beauregard, Serge Goudreau et al., loc. cit.

10 Philippe Jarnoux, «La colonisation de la seigneurie de Batiscan aux 17e et 18e siècles: l'espace et les hommes», Revue d'histoire de l'Amérique française, 40,2 (automne 1986): 163191 .

11 James E. Vance Jr, «California and the Search for the Ideal», Annals of American Geographers, 62,2 (1972): 185-210.
} 
S'agissant des lieux de provenance à l'extérieur du Saguenay, on note que les deux tiers environ de ces familles pionnières - compte tenu de la distribution des «indéterminés» - provenaient de Charlevoix, une relation très exclusive s'établissant entre les deux régions ${ }^{12}$ (Tableau 8). Nos données indiquent également que les immigrants en provenance d'autres régions que Charlevoix manifestaient une préférence pour l'ouest du Saguenay (soit la sous-région du Lac Saint-Jean). On s'attendrait donc à ce que la population de ces paroisses montre une certaine hétérogénéité, ce qui est attesté par le tableau 9. Le bassin patronymique des vieilles paroisses de l'est, comme Anse-Saint-Jean ou Saint-Fulgence, se signale en effet par sa grande homogénéité, caractéristique qui tend à décroître à mesure qu'on s'en éloigne Saint-Ambroise faisant encore une fois exception.

La grande homogénéité de cette population fondatrice semble être une caractéristique essentielle qui la démarque nettement des autres populations pionnières canadiennes-anglaises et américaines à la même époque. Certes, à l'échelle micro-locale, on trouve aussi dans ces dernières populations des exemples de créations homogènes ${ }^{13}$. On a également montré que certains «îlots» socio-culturels pouvaient conserver longtemps leurs traits distinctifs ${ }^{14}$. Mais dans les grandes lignes, et en regard de ce qu'on observe au Saguenay, dans le Bas-Saint-Laurent ou en Mauricie, il est incontestable que la frontière anglo-saxonne offre l'image d'une très grande diversité, en termes de lieux de provenance, d'appartenances ethniques et d'allégeances religieuses ${ }^{15}$. On soupçonne que ces paramètres initiaux ont favorisé la constitution de sociétés très différentes. Sur le plan culturel en particulier, on se trouve en présence de deux problématiques et de deux itinéraires collectifs tout à fait opposés. Dans un cas, on est amené à rechercher les facteurs qui vont introduire la différenciation socio-culturelle; dans l'autre, l'atten-

12 Entre 1840 et 1869 , plus de $80 \%$ des immigrants au Saguenay provenaient de Charlevoix. Gérard Bouchard, «Le peuplement blanc», loc. cit.

13 Par exemple, certaines communautés côtières de Terre-Neuve, comme Goosberry ou Bonavista Bay. Voir John J. Mannion, ed., W. G. Handcock and al., op. cit., 112-113, 128 et ss.

14 Jon Gjerde, «The Effects of Community on Migration: Three Minnesota Townships 1885-1905», loc. cit.; John G. Rice, «The Role of Culture and Community in Frontier Prairie Farming", loc. cit.

15 Á l'appui de cet énoncé, nous renvoyons le lecteur aux excellentes reconstitutions monographiques qui ont été effectuées, notamment, sur le canton d'Euphrasia en Ontario, Darrel A. Norris, «Migration, Pioneer Settlement, and the Life Course: the First Families of an Ontario Township», loc. cit. Sur le North-Dakota, John Hudson, «Two Dakota Homestead Frontiers», Annals of American Geographers, 63,4 (December 1973): 442-462; «Migration to an American Frontier», loc. cit. Sur le Kansas, James C. Malin, «Local Historical Studies and Population Problems», in C. F. Ware, ed., The Cultural Approach to History (Port Washington, N. Y., Kennikat Press Inc., 1940), 300-307. Sur les États du nord des États-Unis, Richard A. Easterlin, George Alter, Gretchen Condran, «Farms and Farm Families in Old and New Areas: the Northern States in 1860», in T. K. Hareven and Maris A. Vinoskis, eds., Family and Population in Nineteenth Century America (1978), 22-85. 


\section{TABLEAU 8}

Lieux de provenance des couples mariés à l'extérieur du Saguenay

\begin{tabular}{|c|c|c|c|c|c|c|c|c|c|c|c|c|c|c|c|c|c|c|}
\hline \multirow{3}{*}{ Paroisses } & \multicolumn{18}{|c|}{ Lieux de provenance } \\
\hline & \multicolumn{2}{|c|}{ Charlevoix } & \multicolumn{2}{|c|}{$\begin{array}{l}\text { Côte de } \\
\text { Beaupré }\end{array}$} & \multicolumn{2}{|c|}{$\begin{array}{l}\text { Région de } \\
\text { Québec }\end{array}$} & \multicolumn{2}{|c|}{ Côte-du-Sud } & \multicolumn{2}{|c|}{$\begin{array}{l}\text { Bas-St- } \\
\text { Laurent }\end{array}$} & \multicolumn{2}{|c|}{ Côte-Nord } & \multicolumn{2}{|c|}{ Mauricie } & \multicolumn{2}{|c|}{$\begin{array}{c}\text { Autres } \\
\text { provenances }\end{array}$} & \multicolumn{2}{|c|}{ Total } \\
\hline & $\mathrm{NA}$ & $\%$ & $\mathrm{NA}$ & $\%$ & $\mathrm{NA}$ & $\%$ & $\mathrm{NA}$ & $\%$ & $\mathrm{NA}$ & $\%$ & $\mathrm{NA}$ & $\%$ & $\mathrm{NA}$ & $\%$ & NA & $\%$ & $\mathrm{NA}$ & $\%$ \\
\hline Anse-St-Jean & 45 & 71,4 & - & - & 1 & 1,6 & 1 & 1,6 & 2 & 3,2 & 2 & 3,2 & - & - & 12 & 19,0 & 63 & 100 \\
\hline St-Fulgence & 59 & 80,8 & 1 & 1,4 & 2 & 2,7 & 1 & 1,4 & 1 & 1,4 & - & - & 1 & 1,4 & 8 & 10,9 & 73 & 100 \\
\hline St-Dominique & 66 & 78,6 & - & - & 1 & 1,2 & 1 & 1,2 & - & - & - & - & - & - & 16 & 19,0 & 84 & 100 \\
\hline St-Gédéon & 52 & 73,2 & - & - & 1 & 1,4 & - & - & 2 & 2,8 & - & - & - & - & 16 & 22,5 & 71 & 100 \\
\hline St-Félicien & 31 & 34,1 & 2 & 2,2 & 10 & 11,0 & 6 & 6,6 & 1 & 1,1 & - & - & - & - & 41 & 45,1 & 91 & 100 \\
\hline St-Cyriac & 9 & 42,8 & - & - & 1 & 4,8 & - & - & - & - & 1 & 4,8 & - & - & 10 & 47,6 & 21 & 100 \\
\hline St-Ambroise & 3 & 16,7 & - & - & 3 & 16,7 & - & - & - & - & 4 & 22,2 & - & - & 8 & 44,4 & 18 & 100 \\
\hline St-Coeur-de-Marie & 2 & 33,3 & - & - & - & - & 1 & 16,7 & 1 & 16,7 & - & - & - & - & 2 & 33,3 & 6 & 100 \\
\hline St-Henri-de-Taillon & 8 & 42,1 & - & - & 2 & 10,5 & 2 & 10,5 & - & - & - & - & - & - & 7 & 36,9 & 19 & 100 \\
\hline Val-Jalbert & 3 & 12,0 & - & - & 3 & 12,0 & 1 & 4,0 & - & - & 1 & 4,0 & 1 & 4,0 & 16 & 64,0 & 25 & 100 \\
\hline Total & 278 & 59,0 & 3 & 0,7 & 24 & 5,1 & 13 & 2,7 & 7 & 1,5 & 8 & 1,7 & 2 & 0,4 & 136 & 28,9 & 471 & 100 \\
\hline
\end{tabular}

Note: La catégorie «Autres provenances» regroupe les couples dont nous n'avons pu retracer l'acte de mariage $(\mathrm{N}=136)$. Nous pensons que ces couples provenaient, pour un tiers environ, de l'est du Québec, et pour le reste, de l'ouest ou de l'extérieur du Québec. (SOREP) 


\section{TABLEAU 9}

Statistique des trente patronymes les plus fréquents dans la population régionale et dans les paroisses étudiées

Liste des Fréquences relatives

trente dans l'ensemble de $(1842-1891)$

TREMBL

BOUCHARD $\quad 4,47$

SIMARD $\quad 4,10$

FORTIN $\quad 2,84$

LAVOIE $\quad 2,37$

GAGNÉ

GOTIER

KOTÉ

SAVAR

BOIVIN

BRASSARD

ARVÉ

BERGERON

DESBIENS $\quad 1,27$

LAROUCHE $\quad 1,23$

POTVIN

GODRO

MORIN

PÉRON

VILLENEUVE

MARTEL

DALAIRE

BOUDRO

WELET

BLACKBUR N

MALTAIS

LAPOINTE

TOTAL

(SOREP)
Fréquences relatives dans les paroisses étudiées

\begin{tabular}{|c|c|c|c|c|c|c|c|c|}
\hline $\begin{array}{l}\text { Anse-St-Jean } \\
\text { (1838-1861) }\end{array}$ & $\begin{array}{l}\text { St-Fulgence } \\
\text { (1839-1871) }\end{array}$ & $\begin{array}{l}\text { St-Dominique } \\
\text { (1841-1866) }\end{array}$ & $\begin{array}{l}\text { St-Cyriac } \\
(1864-1885)\end{array}$ & $\begin{array}{l}\text { St-Ambroise } \\
\text { (1869-1903) }\end{array}$ & $\begin{array}{l}\text { St-Gédéón } \\
(1860-1881)\end{array}$ & $\begin{array}{c}\text { Val-Jalbert } \\
(1902-1911)\end{array}$ & $\begin{array}{l}\text { St-Félicien } \\
\text { (1864-1884) }\end{array}$ & $\begin{array}{c}\text { St-Coeur- } \\
\text { de-Marie } \\
\text { (1879-1889) }\end{array}$ \\
\hline
\end{tabular}

$\mathrm{NA} \quad \% \quad \mathrm{NA} \quad \% \quad \mathrm{NA} \quad \% \quad \mathrm{NA} \quad \%$

NA $\%$

NA $\%$

NA $\%$

$\mathrm{NA} \quad \% \quad \mathrm{NA} \quad \% \quad \mathrm{NA} \quad \%$


tion est d'abord sollicitée par les mécanismes de l'intégration, par les facteurs éventuels de consensus.

L'énoncé qui précède appelle deux remarques. D'abord on pourrait penser que la façon dont les dix paroisses ont été choisies accentue l'homogénéité de ce peuplement. Nous avons en effet retenu, pour les fins de ce projet de recherche, des paroisses comptant parmi les moins touchées par des remaniements territoriaux. Néanmoins, ces paroisses ont connu une croissance aussi rapide que les autres, du moins durant leur période de formation, à laquelle se confine le présent article. Elles ont par conséquent été soumises aux mêmes types d'apports et de renouvellement. Il est utile de préciser par ailleurs que les divisions territoriales n'interviennent jamais avant la création officielle de la paroisse et, sous ce rapport, les paroisses choisies ne se distinguent donc pas des autres. En deuxième lieu, l'homogénéité culturelle que nous observons ici ne doit pas être associée à cohésion et harmonie. Au contraire, comme d'autres, ce genre de société faisait place aux tensions et aux conflits; mais ceux-ci ne prenaient évidemment pas pour fondement ou pour objet des traits que chacun partageait.

\section{3 - PARAMÈTRES SOCIAUX DE L'IMMIGRATION}

À ce stade-ci de la recherche, nous ne disposons pas encore de données sur les causes proprement économiques des migrations (travail en cours). Toutefois, il a été possible d'établir un lien entre l'immigration et la dynamique de la reproduction familiale.

\section{A - L'encadrement familial}

Cette immigration était quasi exclusivement familiale. Selon les données du tableau 10 , près de neuf immigrants sur dix étaient déjà mariés au moment de leur arrivée dans la nouvelle paroisse ${ }^{16}$. On observe peu de variation d'une paroisse à l'autre, sauf pour l'Anse-Saint-Jean et Saint-Fulgence où, au début du peuplement, l'industrie forestière a attiré une main-d'oeuvre saisonnière venue de Charlevoix, dont on perd ensuite la trace. Selon une recherche parallèle conduite au sein de notre centre par Danielle Gauvreau et Mario Bourque (données non publiées), chaque groupe familial impliquait en moyenne sept immigrants ${ }^{17}$. Nous savons par ailleurs que les liens familiaux continuaient de commander étroitement les migrations ultérieures au sein de la région ${ }^{18}$. Du reste,

16 En l'absence de recensements annuels, ce genre de proportion est toujours très difficile à établir. Le chiffre présenté ici est cependant très fiable, les sources dont nous disposions ayant permis d'identifier presque tous les immigrants isolés (proportion d'indéterminés $=5,9 \%$ ).

17 Le groupe familial est défini au sens le plus strict, par référence aux apparentés du premier degré seulement (toutes relations parent-enfant et entre germains). Il inclut aussi le conjoint ou la conjointe.

18 Gérard Bouchard et Jeannette Larouche, «Paramètres sociaux de la reproduction familiale au Saguenay, 1842-1911», Sociologie et sociétés, 19,1 (avril 1987): 133-144. 
TABLEAU 10

État matrimonial des pionniers au moment

de leur arrivée dans la paroisse

\begin{tabular}{|c|c|c|c|c|c|c|c|c|c|c|c|c|c|c|c|c|}
\hline \multirow{3}{*}{$\begin{array}{l}\text { Paroisses } \\
\text { étudiées }\end{array}$} & \multirow{2}{*}{\multicolumn{2}{|c|}{ Mariés }} & \multirow{2}{*}{\multicolumn{2}{|c|}{$\begin{array}{c}\text { Veufs } \\
\text { M }\end{array}$}} & \multirow{2}{*}{\multicolumn{2}{|c|}{$\begin{array}{c}\text { (ves) } \\
\text { F }\end{array}$}} & \multicolumn{8}{|c|}{ Célibataires } & \multirow{2}{*}{\multicolumn{2}{|c|}{ TOTAL }} \\
\hline & & & & & & & \multicolumn{2}{|c|}{ TOTAL } & \multicolumn{2}{|c|}{$\mathbf{M}$} & \multicolumn{2}{|c|}{ F } & \multicolumn{2}{|c|}{ TOTAL } & & \\
\hline & NA & $\%$ & NA & $\%$ & NA & $\%$ & NA & $\%$ & NA & $\%$ & NA & $\%$ & NA & $\%$ & NA & $\%$ \\
\hline Anse St-Jean & 156 & 78,4 & 4 & 2,0 & 3 & 1,5 & 7 & 3,5 & 33 & 16,6 & 3 & 1,5 & 36 & 18,1 & 199 & 100 \\
\hline St-Fulgence & 278 & 79,0 & 10 & 2,8 & 13 & 3,7 & 23 & 6,5 & 34 & 9,7 & 17 & 4,8 & 51 & 14,5 & 352 & 100 \\
\hline St-Dominique & 332 & 92,3 & 3 & 0,8 & 3 & 0,8 & 6 & 1,6 & 18 & 5,0 & 4 & 1,1 & 22 & 6,1 & 360 & 100 \\
\hline St-Gédéon & 141 & 73,8 & 3 & 1,6 & 10 & 5,2 & 13 & 6,8 & 29 & 15,2 & 8 & 4,2 & 37 & 19,4 & 191 & 100 \\
\hline St-Cyriac & 192 & 93,2 & 3 & 1,4 & 1 & 0,5 & 4 & 1,9 & 2 & 1,0 & 8 & 3,9 & 10 & 4,9 & 206 & 100 \\
\hline St-Ambroise & 164 & 90,6 & 4 & 2,2 & 3 & 1,7 & 7 & 3,9 & 10 & 5,5 & 一 & - & 10 & 5,5 & 181 & 100 \\
\hline St-Coeur-de-Marie & 108 & 89,4 & 2 & 1,6 & 2 & 1,6 & 4 & 3,2 & 8 & 6,6 & 1 & 0,8 & 9 & 7,4 & 121 & 100 \\
\hline St-Henri-de-Taillon & 140 & 97,2 & - & - & 2 & 1,4 & 2 & 1,4 & 2 & 1,4 & - & - & 2 & 1,4 & 144 & 100 \\
\hline Val-Jalbert & 186 & 90,7 & 4 & 2,0 & 3 & 1,4 & 7 & 3,4 & 10 & 4,9 & 2 & 1,0 & 12 & 5,9 & 205 & 100 \\
\hline TOTAL & 2061 & 86,3 & 37 & 1,6 & 44 & 1,8 & 81 & 3,4 & 195 & 8,2 & 50 & 2,1 & 245 & 10,3 & 2387 & 100 \\
\hline
\end{tabular}

Nombre d'individus dont l'état matrimonial n'a pu être déterminé: 140. (SOREP) 


\section{TABLEAU 11}

Structure de la population (dix paroisses étudiées)

\begin{tabular}{|c|c|c|c|c|c|c|c|c|c|c|}
\hline \multirow{3}{*}{$\begin{array}{l}\text { Paroisses } \\
\text { étudiées }\end{array}$} & \multicolumn{8}{|c|}{ Classes d'âge } & & \\
\hline & \multicolumn{2}{|c|}{$0-9$} & \multicolumn{2}{|c|}{ 10-19 } & \multicolumn{2}{|c|}{ 20-39 } & \multicolumn{2}{|c|}{40 et +} & \multicolumn{2}{|c|}{$\begin{array}{l}\text { Population } \\
\text { totale }\end{array}$} \\
\hline & NA & $\%$ & NA & $\%$ & NA & $\%$ & NA & $\%$ & NA & $\%$ \\
\hline Anse St-Jean & 81 & 33,1 & 41 & 16,7 & 87 & 35,5 & 36 & 14,7 & 245 & 100 \\
\hline St-Fulgence & 100 & 32,4 & 74 & 23,9 & 83 & 26,9 & 52 & 16,8 & 309 & 100 \\
\hline St-Dominique & 125 & 38,1 & 73 & 22,3 & 101 & 30,8 & 29 & 8,8 & 328 & 100 \\
\hline St-Cyriac & 74 & 28,4 & 75 & 28,7 & 71 & 27,2 & 41 & 15,7 & 261 & 100 \\
\hline Péribonka & 92 & 29,1 & 84 & 26,6 & 94 & 29,7 & 46 & 14,6 & 316 & 100 \\
\hline St-Félicien & 189 & 36,9 & 102 & 19,9 & 169 & 33,0 & 52 & 10,2 & 512 & 100 \\
\hline St-Coeur-de-Marie & 108 & 46,6 & 36 & 15,5 & 59 & 25,4 & 29 & 12,5 & 232 & 100 \\
\hline St-Gédéon & 220 & 33,9 & 137 & 21,1 & 209 & 32,2 & 83 & 12,8 & 649 & 100 \\
\hline St-Bruno & 219 & 38,9 & 101 & 17,9 & 178 & 31,6 & 65 & 11,6 & 563 & 100 \\
\hline $\begin{array}{l}\text { St-François-de- } \\
\text { Sales }\end{array}$ & 167 & 35,8 & 102 & 21,9 & 117 & 25,1 & 80 & 17,2 & 466 & 100 \\
\hline Ensemble & 1375 & 35,4 & 825 & 21,3 & 1168 & 30,1 & 513 & 13,2 & 3881 & 100 \\
\hline
\end{tabular}

Source: Recensements nominatifs du gouvernement canadien et des paroisses

Notes: a) À cause de difficultés posées par les unités de recensement, trois paroisses ont dû être remplacées pour les fins de ce calcul (Saint-Ambroise, Saint-Henri, Val-Jalbert).

b) Nous ne connaissons pas l'âge de 49 personnes.

c) Les chiffres relatifs à la population totale ne sont pas directement comparables à ceux apparaissant au tableau 10. Il faut tenir compte ici de l'effet inégal de la mobilité, de la durée de la période de formation des paroisses, de la date des recensements utilisés et des indéterminés.

ce phénomène n'est pas spécifique au Saguenay; bien au contraire, il semble se manifester à des degrés divers dans tous les contextes de peuplement ${ }^{19}$.

19 Les références sont ici nombreuses. On en trouvera une brève présentation dans Gérard Bouchard, «La dynamique communautaire et l'évolution des sociétés rurales québécoises aux 19e et $20 \mathrm{e}$ siècles. Construction d'un modèle», Revue d'histoire de l'Amérique française, 40,1 (été 1986): 51-71. Il convient d'y ajouter Alan G. Brunger, «Geographical Propinquity Among PreFamine Catholic Irish Settlers in Upper Canada», Journal of Historical Geography, 8,3 (1982): 265-282; John C. Hudson, «Migration to an American Frontier», loc. cit.; Robert P. Swierenga, «Dutch Immigrant Demography, 1820-1880», Journal of Family History, 5 (Winter 1980): 390405, selected by Council of Abstracting Services for summary in Sociology Abstracts; Darrel A. Norris, «Migration, Pioneer Settlement, and the Life Course: the First Families of an Ontario Township», loc. cit.; Robert S. Dilley, «Migration and the Mennonites: Nineteenth Century Waterloo County, Ontario», in Donald H. Akenson, ed., Canadian Papers in Rural History (Gananoque, Langdale Press, 1984), 108-129; John W. Adams and Alice Bee Kasakoff, «Migration and the Family in Colonial New England: the View from Genealogies», Journal of Family History (1984): 24-42. 
Dès lors, on ne se surprend pas que ces grappes d'immigrants délimitées par la parenté aient regroupé plusieurs classes d'âge, étalées sur quelques générations. Ainsi, près de la moitié des hommes mariés ont 30 ans et moins, mais $36 \%$ en ont plus de 40 . La structure des âges reflète aussi cet étalement, même si elle est manifestement dominée par les classes jeunes (Tableau 11). De même, deux fois sur trois, l'immigration impliquait des jeunes couples ayant deux enfants ou moins, mais une part importante des familles $(18,4 \%)$ avaient cinq enfants et plus (Tableau 12). Il serait incorrect de parler ici de deux modèles migratoires, l'un qui serait caractérisé par de jeunes couples, l'autre par de vieilles familles nombreuses. En fait, on est simplement en présence de familles se trouvant à des stades différents de développement. Il suffit que la décision d'émigrer soit retardée de quelques années pour qu'on observe à l'arrivée de jeunes couples sans ou avec peu d'enfants, au lieu de familles très nombreuses et plus âgées.

Sous le rapport du statut matrimonial des immigrants, le Saguenay paraît se distinguer, surtout par rapport aux États-Unis. Dans le NorthDakota $^{20}$ et la vallée du Haut-Mississippi ${ }^{21}$, la moitié des immigrants sont des célibataires. On trouve une situation analogue dans le comté du Wisconsin étudié par M. Curti ${ }^{22}$. Il semble aussi que le caractère familial de l'immigration soit un peu plus prononcé au Saguenay que dans les environs de Québec, dans le Gouvernement de Trois-Rivières et dans les deux cantons francophones de l'Ontario étudiés par Chad M. Gaffield ${ }^{23}$. Par contre, et bien qu'il faille se méfier un peu de ces comparaisons trop ponctuelles, deux études ontariennes dépeignent des situations tout à fait semblables à celle du Saguenay ${ }^{24}$.

En relation avec cette caractéristique, on observe dans cette région une population un peu plus vieille que dans les études américaines qui servent ici de point de référence. On peut penser qu'à l'échelle des États-Unis, la «frontière» suscitait des migrations plus longues et plus incertaines, impliquant une population plus jeune, mariée ou non.

20 John C. Hudson, «The Study of Western Frontier Populations», in Jerome O. Steffen, ed., The American West: New Perspectives, New Dimensions (University of Oklahoma Press, 1979), 35-60.

21 James W. Oberly, «Fertility, Migration and Land Acquisition in the Settlement of the Upper Mississippi Valley». Texte d'une communication au colloque de la Social Science Association à Saint-Lorris, 1986.

22 Merle Curti, The Making of an American Community (Stanford, California, Stanford University Press, 1959), 483 p.

23 Chad M. Gaffield, «Canadian Families in Cultural Context: Hypotheses from the MidNineteenth Century», Historical Papers/Communications historiques, Saskatoon, 1979.

24 Darrel A. Norris, «Migration, Pioneer Settlement, and the Life Course: the First Families of an Ontario Township», loc. cit.; Alan G. Brunger, «Geographical Propinquity Among PreFamine Catholic Irish Settlers in Upper Canada», Journal of Historical Geography, 8,3 (1982): 265-282. 
TABLEAU 12

Nombre d'enfants par famille, au moment de l'arrivée dans la paroisse

\begin{tabular}{|c|c|c|c|c|c|c|c|c|c|c|c|c|}
\hline \multirow[t]{2}{*}{$\begin{array}{l}\text { Paroisses } \\
\text { étudiées }\end{array}$} & \multicolumn{2}{|c|}{0} & \multicolumn{4}{|c|}{$\begin{array}{c}\text { Nombre d'enfants } \\
\quad 3-4\end{array}$} & \multicolumn{2}{|c|}{$5-6$} & \multicolumn{2}{|c|}{7 et plus } & \multicolumn{2}{|c|}{ TOTAL } \\
\hline & NA & $\%$ & NA & $\%$ & NA & $\%$ & NA & $\%$ & NA & $\%$ & NA & $\%$ \\
\hline Anse St-Jean & 24 & 36,4 & 15 & 22,7 & 11 & 16,7 & 14 & 21,2 & 2 & 3,0 & 66 & 100 \\
\hline St-Fulgence & 61 & 57,0 & 23 & 21,5 & 14 & 13,1 & 5 & 4,7 & 4 & 3,7 & 107 & 100 \\
\hline St-Dominique & 46 & 37,1 & 45 & 36,3 & 18 & 14,5 & 9 & 7,3 & 6 & 4,8 & 124 & 100 \\
\hline St-Gédéon & 63 & 44,7 & 38 & 27,0 & 20 & 14,2 & 16 & 11,3 & 4 & 2,8 & 141 & 100 \\
\hline St-Félicien & 41 & 31,8 & 38 & 29,4 & 24 & 18,6 & 17 & 13,2 & 9 & 7,0 & 129 & 100 \\
\hline St-Cyriac & 25 & 30,8 & 18 & 22,2 & 10 & 12,4 & 18 & 22,2 & 10 & 12,4 & 81 & 100 \\
\hline St-Ambroise & 31 & 46,3 & 10 & 14,9 & 14 & 20,9 & 4 & 6,0 & 8 & 11,9 & 67 & 100 \\
\hline St-Coeur-de-Marie & 10 & 19,2 & 15 & 28,9 & 13 & 25,0 & 8 & 15,4 & 6 & 11,5 & 52 & 100 \\
\hline St-Henri-de-Taillon & 20 & 41,7 & 10 & 20,8 & 8 & 16,7 & 4 & 8,3 & 6 & 12,5 & 48 & 100 \\
\hline Val-Jalbert & 27 & 39,2 & 22 & 31,9 & 7 & 10,1 & 7 & 10,1 & 6 & 8,7 & 69 & 100 \\
\hline OTAL & 348 & 39,4 & 234 & 26,5 & 139 & 15,7 & 102 & 11,5 & 61 & 6,9 & 884 & 100 \\
\hline
\end{tabular}

Note: Nombre de familles dont on ignore le nombre d'enfants: 217. (SOREP) 


\section{TABLEAU 13}

\section{Catégories socio-professionnelles}

\begin{tabular}{|c|c|c|c|c|c|c|c|c|c|c|c|c|c|c|c|c|c|c|c|}
\hline \multirow[t]{2}{*}{$\begin{array}{l}\text { Paroisses } \\
\text { étudiées }\end{array}$} & \multicolumn{2}{|c|}{$\begin{array}{l}\text { Industriels } \\
\text { et } \\
\text { Commerçants }\end{array}$} & \multicolumn{2}{|c|}{$\begin{array}{l}\text { Professions } \\
\text { libérales }\end{array}$} & \multicolumn{2}{|c|}{$\begin{array}{l}\text { Cols blancs } \\
\text { spécialisés }\end{array}$} & \multicolumn{2}{|c|}{$\begin{array}{l}\text { Employés de } \\
\text { bureau. Cols } \\
\text { blancs semi } \\
\text { et non spéc. }\end{array}$} & \multicolumn{2}{|c|}{ Cultivateurs } & \multicolumn{2}{|c|}{ Artisans } & \multicolumn{2}{|c|}{$\begin{array}{l}\text { Ouvriers } \\
\text { spécialisés. } \\
\text { Gens de } \\
\text { métier. }\end{array}$} & \multicolumn{2}{|c|}{$\begin{array}{l}\text { Ouvriers } \\
\text { semi et non } \\
\text { spécialisés }\end{array}$} & \multicolumn{2}{|c|}{ TOTAL } & \multirow[t]{2}{*}{$\begin{array}{l}\text { Indé- } \\
\text { ter- } \\
\text { minés }\end{array}$} \\
\hline & $\mathrm{NA}$ & $\%$ & $\mathrm{NA}$ & $\%$ & NA & $\%$ & $\mathrm{NA}$ & $\%$ & $\mathrm{NA}$ & $\%$ & $\mathrm{NA}$ & $\%$ & NA & $\%$ & $\mathrm{NA}$ & $\%$ & NA & $\%$ & \\
\hline Anse St-Jean & 1 & 1,2 & - & - & - & - & - & - & 50 & 61,8 & 1 & 1,2 & 2 & 2,5 & 27 & 33,3 & 81 & 100 & 34 \\
\hline St-Fulgence & - & - & - & - & - & - & - & - & 84 & 76,4 & 2 & 1,8 & 8 & 7,3 & 16 & 14,5 & 110 & 100 & 84 \\
\hline St-Dominique & - & - & - & - & - & - & - & - & 93 & 93,0 & - & - & 2 & 2,0 & 5 & 5,0 & 100 & 100 & 107 \\
\hline St-Félicien & 2 & 1,1 & - & - & - & - & - & - & 170 & 96,7 & 2 & 1,1 & - & - & 2 & 1,1 & 176 & 100 & 81 \\
\hline St-Cyriac & - & - & - & - & - & - & 1 & 1,3 & 73 & 94,8 & - & - & - & - & 3 & 3,9 & 77 & 100 & 39 \\
\hline St-Ambroise & - & - & - & - & - & - & - & - & 74 & 96,1 & - & - & 1 & 1,3 & 2 & 2,6 & 77 & 100 & 30 \\
\hline St-Coeur-de-Marie & - & - & - & - & - & - & - & - & 29 & 87,9 & - & - & - & - & 4 & 12,1 & 33 & 100 & 47 \\
\hline St-Henri-de-Taillon & - & - & - & - & - & - & - & - & 48 & 98,0 & - & - & - & - & 1 & 2,0 & 49 & 100 & 48 \\
\hline Val-Jalbert & 3 & 4,1 & 1 & 1,4 & 7 & 9,6 & - & - & 5 & 6,8 & 2 & 2,7 & 7 & 9,6 & 48 & 65,8 & 73 & 100 & 47 \\
\hline TOTAL & 6 & 0,7 & 2 & 0,2 & 7 & 0,8 & 1 & 0,1 & 731 & 82,4 & 8 & 0,9 & 21 & 2,4 & 111 & 12,5 & 887 & 100 & 602 \\
\hline
\end{tabular}




\section{$B$ - L'établissement des enfants}

Nous avons exposé ailleurs l'idée que ces mouvements migratoires vers le Saguenay entraient dans des stratégies de reproduction familiale dans un cadre rural. La terre arable se faisant rare dans la région de Charlevoix, les familles émigraient soit vers les villes (Québec, Montréal, Nouvelle-Angleterre), soit vers l'intérieur, en direction des régions neuves, pour y établir leurs enfants sur des terres. Dans ce cas, elles liquidaient le bien qu'elles pouvaient posséder, en vue d'acquérir une superficie plus étendue en zone de colonisation. Le nombre d'enfants à établir était à l'origine de cette opération en même temps qu'il en fournissait les moyens: ce sont en effet les enfants, et surtout les fils, qui

TABLEAU 14

Rapport de masculinité selon le groupe d'âge (dix paroisses de l'échantillon)

\begin{tabular}{|l|c|c|c|c|c|}
\hline \multirow{2}{*}{$\begin{array}{l}\text { Paroisses } \\
\text { étudiées }\end{array}$} & \multicolumn{5}{|c|}{ Groupes d'âge } \\
\cline { 2 - 6 } & $0-9$ & $10-19$ & $20-39$ & $40+$ & Ensemble \\
\hline Anse St-Jean & 92,9 & 115,8 & 117,5 & 157,1 & 113,0 \\
\hline St-Fulgence & 122,2 & 131,3 & 124,3 & 126,1 & 125,5 \\
\hline St-Dominique & 155,1 & 108,6 & 140,5 & 141,7 & 137,7 \\
\hline St-Cyriac & 138,7 & 114,3 & 86,8 & 127,8 & 113,9 \\
\hline Péribonka & 104,4 & 115,4 & 108,9 & 119,0 & 110,7 \\
\hline St-Félicien & 94,8 & 137,2 & 111,3 & 116,7 & 109,8 \\
\hline St-Coeur-de-Marie & 100,0 & 125,0 & 90,3 & 141,7 & 105,3 \\
\hline St-Gédéon & 122,2 & 98,6 & 122,3 & 102,4 & 114,2 \\
\hline St-Bruno & 100,9 & 146,3 & 107,0 & 109,7 & 110,9 \\
\hline St-François-de- & 135,2 & 148,8 & 116,7 & 135,3 & 133,0 \\
Sales & & & & & 116,9 \\
\hline Ensemble & 114,2 & 123,0 & 113,5 & 123,0 & \\
\hline
\end{tabular}

Source: Recensements nominatifs du gouvernement fédéral et des paroisses.

Notes: $\quad$ a) À cause des difficultés posées par les unités de recensement, trois paroisses ont dû être remplacées pour les fins de ce calcul (Saint-Ambroise, Saint-Henri, Val-Jalbert). (SOREP)

b) Nous ne connaissons pas l'âge de 49 personnes. 
constituaient la main-d'oeuvre nécessaire aux défrichements ${ }^{25}$. Les familles pionnières étudiées ici paraissent se conformer à ce modèle. Selon le tableau 13, plus de quatre immigrants sur cinq étaient cultivateurs au moment de leur arrivée. Presque tous les autres étaient journaliers; et nous savons que, parmi ces derniers, environ la moitié se sont plus tard établis comme cultivateurs ${ }^{26}$. On remarque par ailleurs un rapport de masculinité relativement élevé (Tableau 14) qui atteste - surtout pour le groupe des 10-19 ans - que le nombre des garçons pesait d'une façon particulière dans la décision d'émigrer.

En conséquence, ce ne sont pas nécessairement les familles les plus pauvres qui émigraient vers les régions de colonisation ${ }^{27}$. On cherchait souvent à étendre le bien familial de manière à ménager aux enfants des conditions de vie au moins comparables à celles de leurs parents ${ }^{28}$.

\section{CONCLUSION}

Les données qui précèdent sont tirées d'un projet de recherche en cours. Elles seront bientôt complétées par des analyses portant cette fois sur les relations de parenté, les caractéristiques économiques, le degré d'enracinement ou de remplacement des populations paroissiales, la descendance des familles pionnières. D'ores et déjà, elles fournissent des éclairages utiles sur la façon d'appréhender certains problèmes. Ainsi, les comparaisons effectuées donnent à croire que, du point de vue des paramètres sociaux considérés (encadrement et reproduction familiale), la société rurale saguenayenne se constitue dans un contexte et selon des modalités relativement semblables à ce qu'on peut observer ailleurs au Québec, en Ontario ou aux États-Unis ${ }^{29}$. Du point de vue culturel cependant, on observe un contraste des plus frappants entre l'extrême homogénéité du peuplement saguenayen — sans doute à

25 Gérard Bouchard, «Family Structures and Geographic Mobility at Laterrière, 1851-1935», The Journal of Family History, 2,4 (Winter 1977): 350-369; «Les systèmes de transmission des avoirs familiaux et le cycle de la société rurale au Québec, du XVIIle au XXe siècle», Histoire sociale/Social History, 16,31 (mai 1983): 35-60; Gérard Bouchard et Isabelle de Pourbaix, «Individual and Family Life Courses in the Saguenay Region, Québec, 1842-1911», Journal of Family History, 12,1-3 (1987): 225-242.

26 Rappelons que R. A. Easterlin et al., «Farms and Farm Families...» ont soutenu une argumentation semblable.

27 C'est une idée qu'on trouve déjà chez Léon Gérin. Voir Gérard Bouchard, «Family Structures and Geographic Mobility at Laterrière, 1851-1935», loc. cit.

28 Encore une fois, le contexte américain offre de nombreux exemples analogues; voir en particulier J. W. Adams, Alice Bee Kasakoff, «Migration and the Family in Colonial New England: the View from Genealogies», loc. cit., et Jon Gjerde, From Peasants to Farmers. The Migration from Balestrand, Norway, to the Upper Middle West (Cambridge, Cambridge University Press, 1985), $319 \mathrm{p}$.

${ }_{29}$ De même, on pourrait aisément montrer - la place ne le permettait pas ici - que les comportements démographiques dans les régions du Québec, notamment au chapitre de la fécondité et de la mobilité, ne diffèrent pas autant qu'on le croit des autres populations pionnières. 
l'image d'autres régions de colonisation du Québec? - et la multiciplicité linguistique et religieuse caractérisant les courants migratoires canadiens-anglais et américains. Pour l'historiographie, comme pour la sociologie des collectivités concernées, ce contraste donne lieu à deux problématiques divergentes qui devraient désormais nourrir toute comparaison: d'un côté, par quels mécanismes et à quel rythme la société homogène va-t-elle opérer sa diversification? De l'autre, comment une culture, une identité va-t-elle s'édifier à partir de la multiplicité? La première question est à l'ordre du jour de nos travaux. Quant à la deuxième, elle a inspiré aux États-Unis une longue tradition historiographique centrée sur le thème de la frontière et d'une nouvelle démocratie ${ }^{30}$. On connaît aussi la thèse du «melting pot», qui est présentement l'objet d'importantes révisions ${ }^{31}$.

Pour le reste, les comparaisons à venir devraient encore faire ressortir, au chapitre des ressemblances, le rôle central de la religion dans la constitution de ces nouvelles sociétés, la règle presque générale de l'exploitant propriétaire, la place longtemps prépondérante de la ferme familiale et des solidarités communautaires. Au chapitre des différences, en ce qui concerne les sociétés anglo-saxonnes, surtout américaines, il faut mentionner: les migrations très longues, la croissance brutale des populations, les dissensions inter-ethniques, les conflits armés avec les Indiens.

Enfin, pour en revenir au cas saguenayen, il est utile de rappeler le clivage existant entre les paroisses du Bas et du Haut-Saguenay, et celles du Lac Saint-Jean. La différence observée dans les lieux de provenance des immigrants à l'est et à l'ouest est en effet une donnée importante, en relation avec le processus de diversification collective. Cette différence se traduit du reste aujourd'hui aussi bien dans la génétique (distribution spatiale de certains gènes délétères) que dans la culture.

30 L'étude de H. S. Barron en offre un très bel exemple. Hal S. Barron, op. cit. Voir aussi, dans la même perspective, Robert D. Mitchell, «The Formation of Early American Cultural Regions: an Interpretation», in James R. Gibson, ed., European Settlement and Development in North American (Toronto, University of Toronto Press, 1978), 66-90.

31 Un courant de recherche récent remet à l'honneur les minorités ethniques et met en lumière leur survivance. Cette résurgence des cultures locales, source d'une diversité durable, est reflétée dans des travaux comme ceux de J. G. Rice, «The Role of Culture and Community in Frontier Prairie Farming», loc. cit. D. Aidan McQuillan, «Territory and Ethnic Identity: Some New Measures of an Old Theme in the Cultural Geography of the United States», in James R. Gibson, European Settlement and Development in North America (Toronto, University of Toronto Press, 1978), 136-169; Robert P. Swierenga, «Dutch Immigration Patterns in the Nineteenth and Twientieth Centuries», in R. P. Swierenga, ed., The Dutch in America: Immigration, Settlement, and Cultural Change (New Brunswick, N. J., Rutgers University Press), 15-42; Walter D. Kamphoefner, «The German Agricultural Frontier: Crucible or Cocoon», Ethnic Forum, 4,1-2 (1984): 21-35, etc. 